\title{
Biocompatible fluid for PD—hanging in the balANZ?
}

Results from the balANZ trial, which compared biocompatible and conventional fluid in patients on peritoneal dialysis, showed no difference in the rate of renal function decline between the groups over the 2-year follow-up. However, secondary outcomes such as time to anuria and time to peritonitis were significantly longer in the biocompatible group, which has important implications for clinical practice.

Biocompatible dialysis fluids have a neutral $\mathrm{pH}$, are lactate-buffered and low in potentially nephrotoxic glucose degradation products. Previous small randomized, controlled trials assessing the effects of biocompatible solutions on residual renal function have yielded conflicting results and have been limited by inadequate statistical power, low event rates, poor methodological quality and single-center designs. "The impetus for conducting the balANZ study was provided by the publication of the Euro-Balance trial," says researcher David Johnson. "The impressive shortterm results raised the possibility that biocompatible peritoneal dialysis fluid solutions might have a clinically important, beneficial effect on residual renal function."
In the balANZ study, the investigators randomly assigned 185 patients from 16 centers across Australia, New Zealand and Singapore to biocompatible $(n=92)$ or control $(n=93)$ fluid. The decline in residual renal function per month in the first year was $-0.22 \mathrm{ml} / \mathrm{min} / 1.73 \mathrm{~m}^{2}$ in the biocompatible group compared with $-0.28 \mathrm{ml} / \mathrm{min} / 1.73 \mathrm{~m}^{2}$ in the control group. In the second year, the difference in glomerular filtration rate decline between the treatment groups was even smaller $\left(0.01 \mathrm{ml} / \mathrm{min} / 1.73 \mathrm{~m}^{2}\right.$ per month).

\section{$\mathbf{4}$...the number of patients experiencing peritonitis was lower in the biocompatible} group... 77

Time to anuria was significantly longer in the biocompatible group than in the control group and anuria developed in fewer patients in the biocompatible group than in the control group (7\% versus $20 \%$, respectively). Systolic and diastolic blood pressure and serum albumin levels were significantly lower in the biocompatible group at 24 months but not at other time points, whereas other indices of fluid status, such as serum sodium concentration and hemoglobin levels, were similar throughout.

Importantly, the number of patients experiencing peritonitis was lower in the biocompatible group than in the control group (30\% versus $49 \%$, respectively) and time to first peritonitis episode was also significantly longer in the biocompatible group. Technique survival and patient survival did not differ between the two groups.

"In terms of future research, we are undertaking several studies, including post hoc analyses of the balANZ trial with respect to the impact of biocompatible fluid administration on the microbiology, treatment and outcomes of peritonitis episodes, as well as on peritoneal membrane function," says Johnson. "In addition, we will conduct a study of renal, peritoneal membrane and inflammatory biomarkers from plasma, urine and dialyzate effluent collected during the balANZ trial, which will be correlated with clinical outcomes."

Helene Myrvang

Original article Johnson, D. W. et al. Effects of biocompatible versus standard fluid on peritoneal dialysis outcomes. J.Am. Soc. Nephrol. doi:10.1681/ ASN.2011121201 